University of Nebraska - Lincoln

DigitalCommons@University of Nebraska - Lincoln

20th \& 21st Century French and Francophone Modern Languages and Literatures, Department Studies International Colloquium

3-16-2020

Narrateur, narration et roman à thèse dans Sérotonine

Gerald Prince

University of Pennsylvania, gprince@babel.ling.upenn.edu

Follow this and additional works at: https://digitalcommons.unl.edu/ffsc2020

Part of the Comparative Literature Commons, French and Francophone Literature Commons, and the Other French and Francophone Language and Literature Commons

Prince, Gerald, "Narrateur, narration et roman à thèse dans Sérotonine" (2020). 20th \& 21st Century French and Francophone Studies International Colloquium. 4.

https://digitalcommons.unl.edu/ffsc2020/4

This Article is brought to you for free and open access by the Modern Languages and Literatures, Department of at DigitalCommons@University of Nebraska - Lincoln. It has been accepted for inclusion in 20th \& 21st Century

French and Francophone Studies International Colloquium by an authorized administrator of DigitalCommons@University of Nebraska - Lincoln. 


\section{Narrateur, narration et roman à thèse dans Sérotonine}

Gerald Prince, University of Pennsylvania

20th \& 21st Century French and Francophone Studies International Colloquium, University of Nebraska-Lincoln, March 26-28, 2020

Le narrateur autodiégétique de Sérotonine, on l'a déjà souvent remarqué, fait le portrait d'une dépression (la sienne) et oppose tradition et modernité, agriculture et industrie, campagne et ville, amour ou sexe (c'est, semble-t-il, un peu la même chose) et solitude ou séparation. II souligne également les multiples formatages dont nous somme victimes, les nombreux mécanismes qui nous emprisonnent, tout en admettant que nous ne sommes pas entièrement déterminés et tout en détaillant une imposante série d'occasions perdues (qu'il s'agisse de proposer le mariage à la femme qu'on aime, de lui expliquer une infidélité ou de se tuer dans un accident de voiture).

Agé de quarante-six ans, veule, dépressif, impuissant, le narrateur est ingénieur agronome (spécialisation d'écologie). Après l'Agro, il a travaillé pour Monsanto puis pour le ministère de l'Agriculture. Il s'appelle Florent-Claude Labrouste et déteste son prénom, qui convient à sa situation sinon à son apparence. La combinaison Florent-Claude lui paraît ridicule (il préfère son second prénom, Pierre) et, comme il le dit lui-même, Florent, qui évoque les fleurs, "est trop doux, trop proche du féminin Florence [et] ne correspond nullement à [s]on visage aux traits énergiques." Claude fait penser aux Claudettes, les danseuses de Claude 
François, mais aussi aux reines-claudes et à la claudication. Quant au nom "Labrouste", il connote la brousse et le broutage. D'autres appellations dans Sérotonine ne sont pas moins parlantes. C'est ainsi que "Kate", le nom du premier amour du protagoniste, veut dire "pure" à I'origine; Camille Da Silva, celui de la femme de sa vie, rappelle le camélia aussi bien que la forêt et renvoie étymologiquement aux jeunes filles nobles qui participaient aux rites sacrés; Aymeric d'Harcourt-Olonde, le meilleur (et seul) ami de Florent-Claude, porte un nom qui remonte au Moyen Age; Cécile, l'épouse de ce nouveau "chevalier" qu'elle abandonne pour un pianiste, s'appelle comme la patronne des musiciens; et le docteur Azote (sans vie), qui consulte rue d'Athènes, est onomastiquement bien approprié.

Florent-Claude Labrouste est un narrateur crédible, aussi crédible, en tout cas, que le sont Google ou Wikipedia. Presque tout ce qu'il dit de la géographie, de l'hôtellerie, de la restauration parisienne ou normande s'avère juste et même les menus auxquels il se réfère correspondent plus ou moins à ceux qui sont présentés sur la Toile (j'ai vérifié). Labrouste est également fiable (dans l'acception que Wayne Booth donne à ce terme). C'est évidemment là, comme toujours, une évaluation pragmatique, mais elle me semble bien fondée car rien dans Sérotonine ne vient contredire ou mettre en question les déclarations du narrateur et, par surcroît, ces déclarations font écho à celles que l'on trouve dans bien d'autres textes de Michel Houellebecq: Extension du domaine de la lutte, Les particules élémentaires, Soumission ... En outre, Labrouste est un narrateur intrusif, pour ne pas dire bavard, qui n'hésite pas à exprimer ce qui lui passe par la tête, à vitupérer contre "le commerce et tout ce qui s'y apparente, l'idée de 'hautes études commerciales' [étant à ses] yeux une profanation de la notion même d'études", à fustiger les Hollandais ("vraiment des putes"), les bourgeois écoresponsables et les 
écologistes radicaux, à soutenir que Niort est "une des villes les plus laides" qui soient ou à se lancer dans des dissertations littéraires ou musicales. Car il est relativement cultivé. Sa narration rappelle parfois saint Paul, Sartre, Flaubert ou Apollinaire, il discute Deep Purple et Pink Floyd, Gogol et Rousseau, Marcel Proust et Thomas Mann; il exécute Christine Angot, Georges Bataille et Maurice Blanchot. D'ailleurs, Labrouste est un narrateur "self-conscious", un narrateur conscient de l'être, qui commente la langue qu'il emploie ('je ne veux pas dire", "je m'exprime mal", "comme on dit") ainsi que le progrès de sa narration ("j'y reviendai", "mais n'anticipons pas", "je m'égare"), qui raconte des histoires à la Theodor Fontane ou à la Segalen, qui imagine des scénarios possibles, pour comédie romantique, par exemple, ou, au contraire, pour film porno et qui recourt fréquemment au disnarré.

Un grand nombre de remarques qu'émet le narrateur sur sa situation, sur sa propre narration ou sur d'autres récits font plus que suggérer pourquoi il raconte. Labrouste est perdu, il s'est perdu, il est effroyablement seul. Il n'a plus de compagne ni de famille ni d'amis, il ne travaille plus, il a de moins en moins d'importance collective diraient Céline ou Sartre, et la réceptionniste de l'hôtel Mercure dans lequel il loge, les serveurs du café O'Jules, les vendeuses du Carrefour City ou encore le docteur Azote finissent par devenir ses principaux interlocuteurs. Raconter lui permet de se sentir plus proche d'autres humains, ne seraient-ce que des lecteurs possibles, et lui permet aussi d'établir certains points de repère comme "de créer une sorte de réalité alternative, de remonter à l'origine d'une bifurcation temporelle, en quelque sorte d'acquérir des crédits de vie supplémentaires." Lui qui avait trop à vivre lorsqu'il "fréquentait" Camille "pour perdre [son] temps en selfies" est pareil à un mort maintenant et ne peut plus que substituer à la vie sa représentation. Il peut au moins établir ainsi un bilan, se convaincre 
qu'il a une histoire et qu'il a vécu. Il peut repenser à certaines heures, éclaircir certains sujets, comprendre certaines actions, "organiser un mini-cérémonial d'adieux autour de sa libido" et revoir les femmes qui l'ont aimé, les femmes qu'il a aimées. Avec Kate, avec Camille, Labrouste n'a pas su exprimer ses sentiments, il n'a pas su s'expliquer et dire tout simplement "je t'aime." Son récit l'aide à le faire ou peut-être même à trouver les mots appropriés. De plus, il l'aide à dénoncer ce qui est inadmissible, à porter témoignage, à étayer ses démonstrations. Enfin, comme raconter est quelque chose qu'il est capable de faire, il le fait.

Que Sérotonine soit une narration à la première personne n'est pas pour surprendre. Confit dans sa solitude, Florent-Claude Labrouste éprouve plus que des embarras à la quitter, à se quitter. Unique issue envisageable, le couple, le rêve à deux, "un homme et une femme seuls face à face." Car le monde social moderne est impitoyable, c'est une machine à détruire l'amour. Le narrateur sent qu'il aurait pu vivre seul avec Camille et être heureux. Mais la vie, comme aussi la veulerie silencieuse du protagoniste, en a décidé autrement. Le récit homodiégétique implique toujours une distance (et donc un isolement), une distance qui peut être insignifiante ou au contraire considérable entre soi et soi, je-narrant et je-narré. Dans le cas de Labrouste, la distance est mise en relief non seulement par sa "self-consciousness", sa conscience d'être un narrateur, mais aussi par son emploi fréquent du discours indirect libre, qui, tout en marquant un certain éloignement, finit par constituer un des seuls moyens qu'il trouve de joindre sa voix à celle d'un autre, par sa mise au même niveau du notable et du banal, de l'impulsif et du raisonné, de l'arbitraire et de l'authentique, ainsi que par sa pratique du second degré et par son humour. Si la vie de Labrouste n'est pas une tragédie, c'est au moins une farce tragique dirait lonesco, et il ne lui paraît guère absurde "de développer une attitude 
humoristique" envers elle et de la considérer "comme une plaisanterie". La distance est également accentuée par le fait que le récit de Labrouste est un récit rétrospectif. Tout (ou presque) est déjà arrivé. S'il ne peut s'empêcher d'"espérer au-delà de toute espérance", le protagoniste n'y peut rien changer. Ce qui est fait est fait, irrémédiablement et pour toujours. Enfin, il convient de noter que ce récit est un récit écrit bien que teinté d'oralité, c'est-à-dire que ce n'est pas exactement un récit spontané.

Mais si, par sa nature, ce récit désigne et constitue une distance, ses visées n'en sont pas moins parfaitement claires. Les antithèses en termes desquelles il se déploie-j'en ai déjà mentionné les plus marquantes-se multiplient et se fortifient les unes les autres. De plus, le récit ne cesse de souligner ce qu'il est ou ce qu'il veut être, ce qu'il n'est pas ou ne veut pas être: ni comédie romantique ou écrit pornographique, certes, ni roman sentimental, non plus, ou scénario de film hollywoodien dans lequel le héros et l'héroïne se retrouvent à la fin, ni drame classique entre amour et devoir, ni récit de filiation psychologique, mais bien plutôt autoportrait et farce tragique, jérémiade et apologie d'un monde en voie de disparition. Les motifs importants-que ce soit le fromage (quoi de plus français!) (et il est en péril), ou les vaches, nourricières, amples, majestueuses, sacrées, ou l'agriculture à l'ancienne ("labourage et pâturage sont les deux mamelles de la France")-viennent renforcer les antithèses, de même que l'espace narratif (la plus grande partie de l'action a lieu dans la Manche, en Normandie, avec ses nouveaux chouans et ses nouveaux Don Quichotte), que les nourritures consommées (cuisine traditionnelle ou conserves, produits régionaux ou fooding novateur) ou que les références artistiques et littéraires. 
Le sens et la fonction de ces références sont d'ailleurs explicitées ou bien faciles à établir. Par exemple, Aymeric et Florent-Claude écoutent Pink Floyd et Ummagumma parce que "le disque à la vache, c'est de circonstance". Bernard Lavilliers est évoqué ("Est-ce ainsi que les hommes vivent") parce qu'il chante le mal-être et l'auto-trahison mais aussi, sans doute, parce qu'il a soutenu les Gilets jaunes. Si Barbey d'Aurevilly est mentionné, c'est que le château d'Aymeric "a servi de cadre à la fin du dernier roman [de l'auteur], Une histoire sans nom" et que I'héroïne de cette histoire, Lasthénie de Ferjol, a donné son nom à un type de pathomimie. Si Nerval est (mal) cité, c'est qu'il est l'auteur des Filles du feu et qu'il s'est suicidé à quarante-six ans. Baudelaire, mort à quarante-six ans lui aussi, Lamartine, avec son romantisme sentimental et mélancolique, Thomas Mann et Marcel Proust, pour qui "toute la culture du monde ne servait à rien" et qui restèrent "prêts à se prosterner devant n'importe quelle chatte humide, ou n'importe quelle jeune bite vaillamment dressée" sont également convoqués. Quant à Conan Doyle, en qui vibre à chaque page "un cœur sincère et bon" et qui apporte à Labrouste "un certain réconfort", il s'est tourné, en fin de compte, vers le spiritisme, "espoir dernier, consolation ultime de tous ceux qui ne parviennent ni à accepter la mort de leurs proches, ni à s'adhérer à la chrétienté". Pour Labrouste également, demeure, en dépit de tout, sinon une espérance, du moins une incertitude, l'idée que "quelque chose dans les cieux va reprendre la main, va décider arbitrairement de distribuer une nouvelle donne, de relancer les dés". Dieu est peut-être "un scénariste médiocre" mais "il nous donne des directives parfois très précises. Ces élans d'amour qui affluent dans nos poitrines jusqu'à nous couper le souffle, ces illuminations, ces extases, inexplicables si l'on considère notre nature biologique, notre statut de simples primates, sont des signes extrêmement clairs". Rien ne compte que le sexe et l'amour, que le 
sexe dans l'amour, et ce dernier "rest[e] la seule chose en laquelle on puisse encore, peut-être, avoir foi".

Au niveau thématique, narrationnel, référentiel, caractériel, c'est la même antienne. Sérotonine adopte la stratégie surdéterminatrice du roman à thèse et la justifie. Je cite, pour terminer, la fin du texte: "Et je comprends, aujourd'hui, le point de vue du Christ, son agacement répété devant l'endurcissement des cœurs: ils ont tous les signes, et ils n'en tiennent pas compte. Est-ce qu'il faut vraiment, en supplément, que je donne ma vie pour ces minables? Est-ce qu'il faut vraiment être, à ce point, explicite? II semblerait que oui." 